\title{
Protons accelerated in the target normal sheath acceleration regime by a femtosecond laser
}

\author{
L. Torrisi,${ }^{1, *}$ M. Cutroneo, ${ }^{2}$ A. Torrisi,${ }^{2}$ L. Silipigni,,${ }^{1}$ G. Costa, ${ }^{1}$ M. Rosinski, ${ }^{3}$ J. Badziak,${ }^{3}$ \\ J. Wołowski, ${ }^{3}$ A. Zaraś-Szydłowska, ${ }^{3}$ and P. Parys ${ }^{3}$ \\ ${ }^{1}$ Dipartimento di Scienze Fisiche-MIFT, Università di Messina, Italy \\ ${ }^{2}$ Nuclear Physics Institute, CAS, Rez, Czech Republic \\ ${ }^{3}$ Institute of Plasma Physics and Laser Microfusion, Warsaw, Poland
}

(Received 15 November 2018; published 8 February 2019)

\begin{abstract}
Advanced targets based on thin films of graphene oxide covered by metallic layers have been irradiated at high laser intensity $\left(\sim 10^{19} \mathrm{~W} / \mathrm{cm}^{2}\right)$ with $40 \mathrm{fs}$ laser pulses to investigate the forward ion acceleration in the target normal sheath acceleration regime. A time-of-flight technique was employed with silicon-carbide detectors and ion collectors as fast on-line plasma diagnostics. At the optimized conditions of the laser focus position with respect to the target surface was measured the maximum proton energy using $\mathrm{Au}$ metallic films. A maximum proton energy of $2.85 \mathrm{MeV}$ was measured using the Au metallization of $200 \mathrm{~nm}$. The presence of graphene oxide facilitates the electron crossing of the foil minimizing the electron scattering and increasing the electric field driving the ion acceleration. The effect of plasma electron density control using the graphene oxide is presented and discussed.
\end{abstract}

DOI: 10.1103/PhysRevAccelBeams.22.021302

\section{INTRODUCTION}

In the ambit of laser-driven ion acceleration in solid targets using the target normal sheath acceleration (TNSA) regime [1], let us remember that energetic ions are accelerated not directly by the laser fields but by the plasma field. The plasma fields are formed due to the laser heated electrons; plasma electrons can mediate the forces of laser fields on ions by generating quasistatic electric fields which rise from a local charge separation generated in the rear side of the laser irradiated foil. The quasistatic field varies on a timescale comparable to the laser pulse duration and can be of the same magnitude as that of the fast oscillating laser fields, giving the ions significantly longer time to accelerate [2]. The laser energy can be transferred to the plasma electrons by various mechanisms leading to the different ion acceleration regime depending on the laser parameters, the irradiation conditions, and the target properties.

The charges generated in the sheath field behind the foil produce an electrical field $\mathrm{E}_{s}$ given by [3]

$$
E_{s}=\sqrt{2} k T / e \lambda_{D}
$$

\footnotetext{
Corresponding author. lorenzo.torrisi@unime.it

Published by the American Physical Society under the terms of the Creative Commons Attribution 4.0 International license. Further distribution of this work must maintain attribution to the author(s) and the published article's title, journal citation, and DOI.
}

where $k T$ is the electron temperature $(\mathrm{eV}), e$ the electron charge and $\lambda_{D}$ the spatial extension of the sheath field. Ions will be accelerated in a direction orthogonally to the target surface with an angular aperture inversely proportional to their mass and charge state. The $\lambda_{D}$ value can be evaluated by the Debye length of fast electrons:

$$
\lambda_{D}=\sqrt{\frac{\varepsilon_{0} k T}{e^{2} n_{e 0}}},
$$

where $\varepsilon_{0}$ is the dielectric constant in vacuum and $\mathrm{n}_{e 0}$ is the maximum electron density of the hot electrons.

By the Poisson equation, it is possible to calculate the maximum electric field of the ion acceleration, which is given by

$$
E_{s}=\sqrt{\frac{2 n_{e 0} k T}{\varepsilon_{0}}},
$$

in agreement with the literature $[3,4]$.

Thus, electron density and plasma temperature play an important role in the determination of the maximum electric field driving the ion acceleration. The electron density can be increased using metallic heavy elements placed at the target surface, such as gold or tantalum, from which electrons can be accelerated by the laser electromagnetic pulse to relativistic velocity in the direction crossing the thin foil thickness. The plasma temperature can be increased not only by the laser intensity and 
wavelength but also by the return electrons on the irradiated target $[1,2]$.

The role of the relativistic electron crossing the foil is of primary importance, to this we proposed to use a foil of graphene oxide $(\mathrm{GO})$ with a micrometric thickness, covered by metallic films, in order to reduce the electron energy loss in the low density foil [5], the electron scattering and the laser reflections. In this way, we expect to enhance the hot electron density in the rear side of the foil and the driving electric field of ion acceleration, as it will be presented and discussed observing the experimental measurements.

\section{MATERIALS AND METHODS}

A femtosecond Ti-sapphire laser system (PULSAR) was used for this experiment at IPPLM laboratory of Warsaw. It operates at $800 \mathrm{~nm}$ wavelength, $40 \mathrm{fs}$ pulse duration, $300 \mathrm{~mJ}$ maximum pulse energy, p-polarized radiation, up to $10^{19} \mathrm{~W} / \mathrm{cm}^{2}$ intensity, with a minimum focal diameter of 10 microns.

The focal position could be moved from -500 microns (in front of the target surface) to $0 \mu \mathrm{m}$ (at the target surface), and up to +500 microns (inside the target surface) using a micrometric step motor controllable in high vacuum $\left(10^{-6}\right.$ mbar). The pulse contrast [prepulses and amplified spontaneous emission (ASE)] was $10^{-8}$. The laser was employed to study the TNSA forward ion acceleration at high intensity when thin foils are irradiated in normal incidence.

Foils of reduced rGO, $7 \mu \mathrm{m}$ thick, were employed to have the following ten advantages [6]: (i) high mechanical resistance of the foil; (ii) high IR laser light transmission; (iii) high electrical conductivity of the foil; (iv) high effect of electron channeling though GO; (v) high hydrogen concentration absorbed by GO; (vi) low laser reflection in $\mathrm{GO}$; (vii) low foil density $\left(1.45 \mathrm{~g} / \mathrm{cm}^{3}\right)$; (viii) low electron stopping power in GO; (ix) low electron scattering in $\mathrm{GO}$; (x) control of the electron plasma density by metals films $(\mathrm{Al}, \mathrm{Cu}$, and $\mathrm{Au})$.

The rGO foil was covered by $200 \mathrm{~nm}$ metallic, in order to increase the number of electrons accelerated in the surface by the laser light and crossing the rGO foil. The laser focalization on the target is accomplished by an optical microscope showing the minimum spot (10 microns in diameter) at high magnification on an LCD screen. Figure 1(a) shows a scheme and a photo (inset) of the experimental setup, Fig. 1(b) a photo of the used rGO and Fig. 1(c) a GO foil covered by $200 \mathrm{~nm}$ $\mathrm{Au}$ film.

$\mathrm{SiC}$ semiconductor detectors and ion collectors (IC) were employed for the fast on-line plasma diagnostics using a time-of-flight (TOF) technique, as in previous experiments [7]. The $\mathrm{SiC}$ detectors have an active depth zone of 80 microns, an active area of $4 \mathrm{~mm}^{2}$ and a surface metallization constituted by $200 \mathrm{~nm} \mathrm{Ni}_{2} \mathrm{Si}$.
$\mathrm{SiC}$ detectors have a $3.3 \mathrm{eV}$ energy gap, thus they detect UV, $x$ rays, energetic electrons and ions but not visible radiations. They are structured such as a Schottky diode with a fast charge collection, high electron mobility, and low reverse current, and show advantages with respect to silicon for applications to plasma diagnostics. The detection efficiency depends on the surface metallization thickness and active depth zone. Figure 2(a) shows typical curves of detection efficiency versus the energy for photons, electrons, protons and gold ions.

Three $\mathrm{SiC}$ detectors were placed at $0^{\circ}, 7^{\circ}$ and $-7^{\circ}$ in the forward direction to a distance of $82 \mathrm{~cm}$ from the target. The central one ( $\mathrm{SiC} 1)$ was employed without absorber and the other two use a Mylar absorber $12 \mu \mathrm{m}$ (SiC2) and $23 \mu \mathrm{m}$ (SiC3) in thickness, respectively. The Mylar absorber thicknesses correspond to 700 and $1080 \mathrm{keV}$ proton range, respectively. The IC detectors were also used in the TOF approach, in the forward direction at $+4^{\circ}$ at a distance of $36 \mathrm{~cm}$. SiC and IC detectors are connected to a fast storage oscilloscope $(500 \mathrm{MHz}, 2.5 \mathrm{GS} / \mathrm{s})$ and are triggered by the photopeak of one $\mathrm{SiC}$ detector.

The ion stopping powers and range in the absorbers were calculated using SRIM code [8].

\section{RESULTS}

The experiment was conducted using a fs laser pulse at high contrast $\left(\sim 10^{-8}\right)$ whose shape and relative intensity vs time are reported in Fig. 2(b).

The spectrum shows a pedestal with a duration of about $0.4 \mathrm{ps}$ with a mean relative intensity, with respect to the main peak assumed 100, of about $10^{-5}$, and the main pulse with a duration (FWHM) of about $40 \mathrm{fs}$ and a relative intensity of 100. This laser peak shape is very important to be sure that the prepulse (pedestal) shape, duration and intensity, is many orders of magnitude lower than the main pulse and cannot generate significant preplasma before the arrival of the main pulse on the target.

A typical SiC-TOF spectrum obtained irradiating a GO foil, 7 microns in thickness, covered by $200 \mathrm{~nm}$ Au film, using maximized conditions of ion acceleration is reported in Fig. 3(a). The conditions of irradiation consist of the use of minimum focal spot size, choice of a flat and less roughness surface, use of a laser pulse energy of $351 \mathrm{~mJ}$, 40 fs pulse duration, p-polarized radiation, and focal position of laser $\mathrm{FP}=+340$ microns, i.e., the laser focus is inside the target and far beyond the thickness of the foil. In such conditions, the spectrum shows a high photopeak due to the detection of $\mathrm{x}$ rays and relativistic electrons, followed by a high and large peak due to the detection of ions. The minimum arrival time of the ion signal is due to the faster protons, which are detected at a TOF of $35 \mathrm{~ns}$, i.e., at a velocity of $2.34 \times 10^{7} \mathrm{~m} / \mathrm{s}$ and a kinetic energy of $2.85 \mathrm{MeV}$. The ion peak indicates that also ions at lower velocity are detected. The ion peak shape indicates that the ion detection is not due only to protons but also to other 

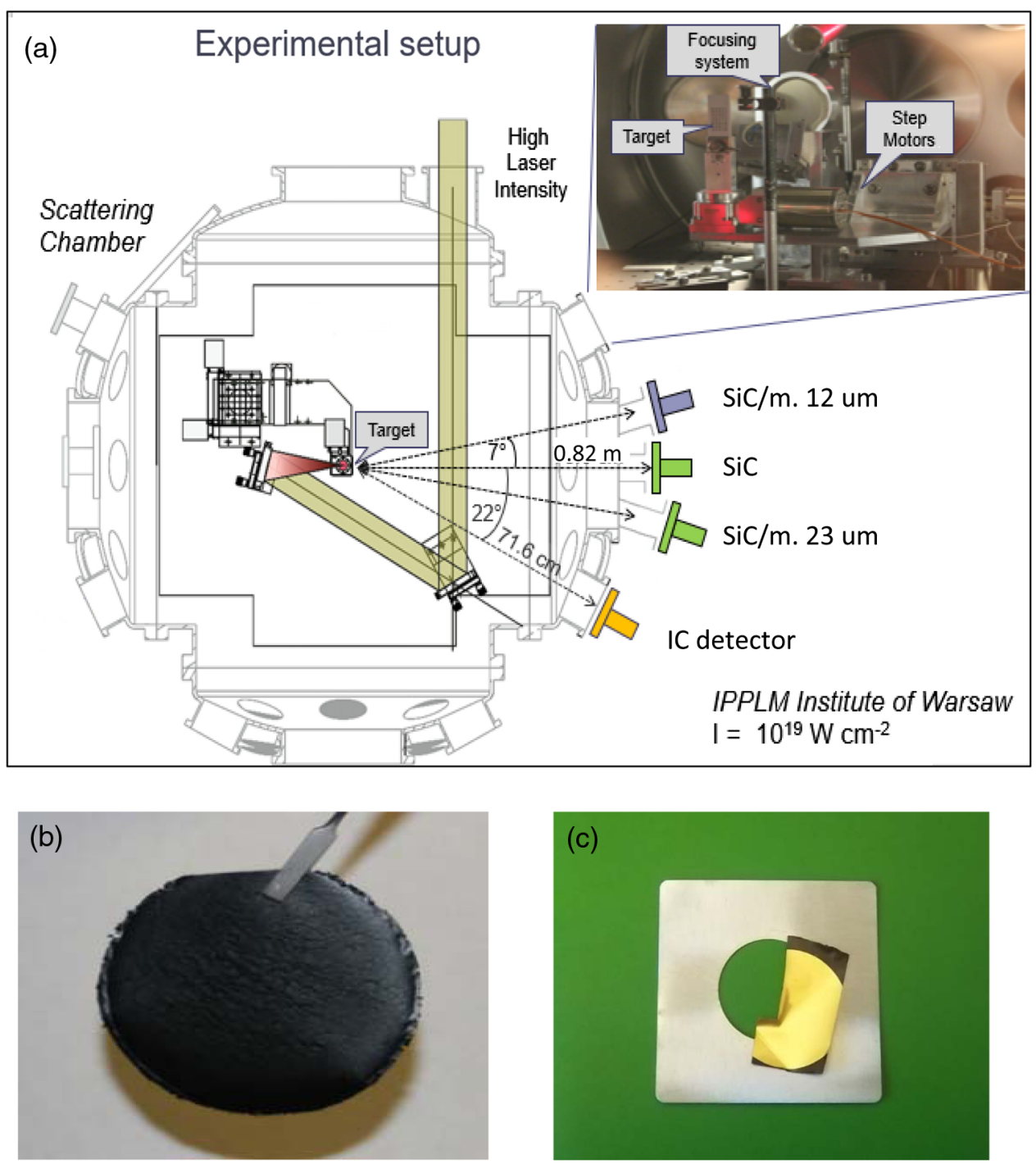

FIG. 1. Scheme and photo (inset) of the experimental setup (a) graphene oxide foil (b) and GO foil covered by $200 \mathrm{nmAu}$ film (c).

light ion species. The peak, in fact, cannot be fitted by a Boltzmann proton distribution, the peak tail would indicate low proton energy not compatible with TNSA acceleration and the peak sawing would not be justifiable with the revelation of a single type of ions. To this, the CoulombBoltzmann-shifted (CBS) distribution [9] was considered assuming that the accelerated ions are due to protons, as foil impurities, and as carbon due to the matrix composition. Oxygen ions could also be present but their concentration would be negligible compared to that of carbon ions, thus they were neglected in the ion peak deconvolution. Assuming that the maximum proton energy is $2.85 \mathrm{MeV}$ and that it is indicative of the maximum acceleration voltage of $2.85 \mathrm{MV} / \mathrm{z}$ (being $\mathrm{z}$ the charge state), the deconvolution presented in Fig. 3(b) is referred to the proton and carbon deconvolution considering the six charge states of carbon ions. The medium energy of the $\mathrm{C}^{6+}$ ions is $2.85 \times 6=17.1 \mathrm{MeV}$, while that of $\mathrm{C}^{1+}$ is $2.85 \mathrm{MeV}$. The TOF shifts of the six charge states of carbon ions indicated an average voltage potential of 1.45 MV. The average Boltzmann distribution of the carbon energy distributions indicates an average plasma temperature of $230 \mathrm{keV}$.

This temperature is in agreement with the theoretical predictions [2]:

$$
k T=m_{0} c^{2}\left(\sqrt{1+\frac{I_{0} \lambda_{L}^{2}}{1.37 \times 10^{18}}}-1\right),
$$

where $m_{0}$ is the rest electron mass, $c$ the light velocity, $I_{0}$ the laser intensity (in $\mathrm{W} / \mathrm{cm}^{2}$ ) and $\lambda_{L}$ the laser light wavelength (in $\mu \mathrm{m}$ units). Because with the used focalization in such measure the laser intensity was about $3 \times 10^{18} \mathrm{~W} / \mathrm{cm}^{2}$ and the used wavelength of 0.8 microns, the theoretical evaluation given by Eq. (4) corresponds to $270 \mathrm{keV}$. 

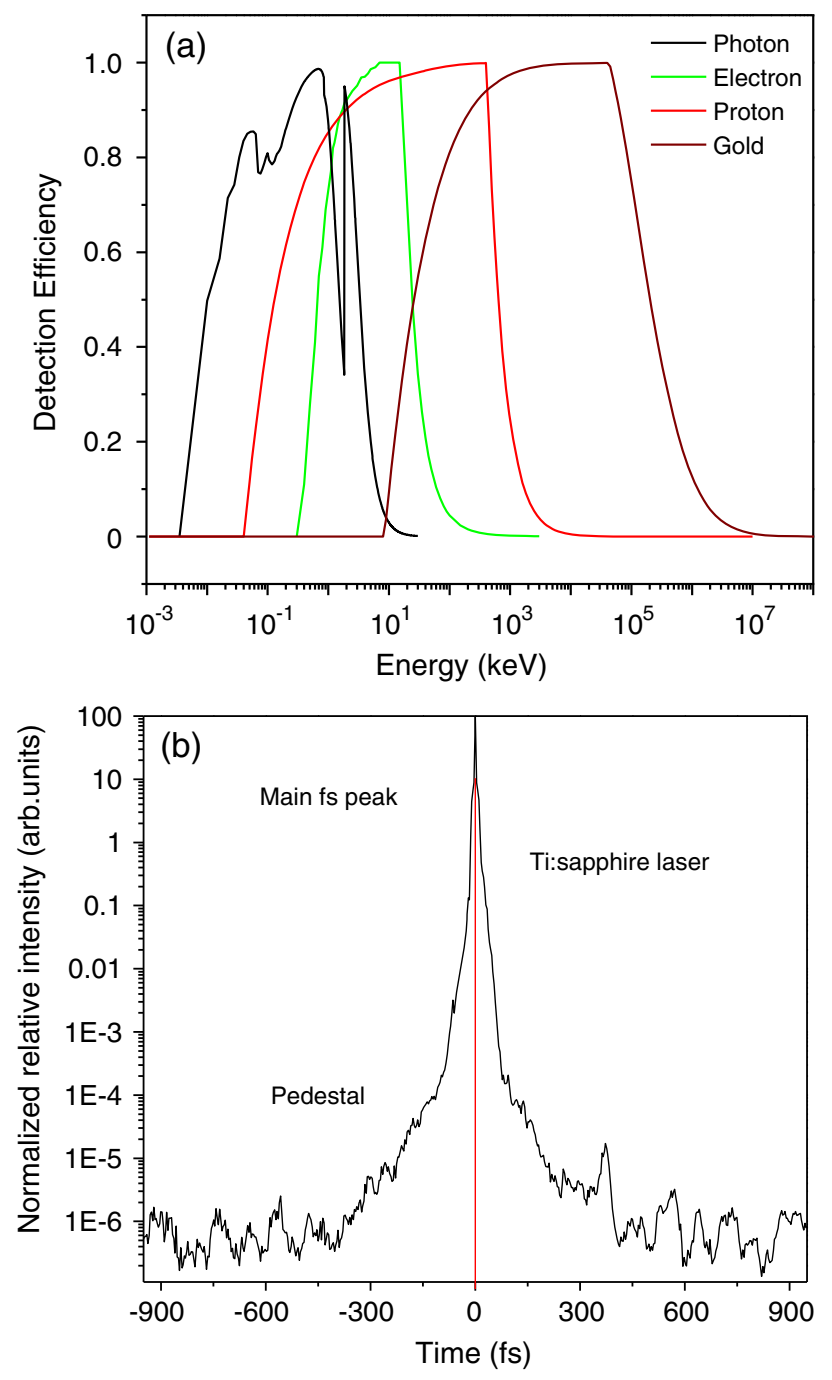

FIG. 2. Detection efficiency as a function of the energy and of the kind of radiation (a) and shape of the fs laser pulse vs time (b).

The adiabatic plasma expansion velocity in the vacuum is calculable at this temperature using the equation [9]

$$
\mathrm{v}=(\gamma \mathrm{kT} / \mathrm{m})^{1 / 2}
$$

where $\gamma$ is the adiabatic coefficient (1.6 for monoatomic species) and $\mathrm{m}$ the ion mass. For carbon ions, representing the major component of the particles emitted from the plasma, the velocity assumes a value of $1.7 \times 10^{6} \mathrm{~m} / \mathrm{s}$.

A similar proton energy was obtained by the IC collector, although the spectrum was more disturbed by electromagnetic noise, and was not reported in this paper.

Figure 3 gives an indication also on the ion current emitted from protons and carbon ions. Although the detection solid angle subtended by the $\mathrm{SiC}$ detector is very little, corresponding to $6 \mu \mathrm{str}$, the electrical signal acquired by the $\mathrm{SiC}$ is of about $2 \mathrm{~V}$ with $10 \mathrm{~ns}$ duration. Due to the input oscilloscope resistance of $50 \Omega$, it corresponds to
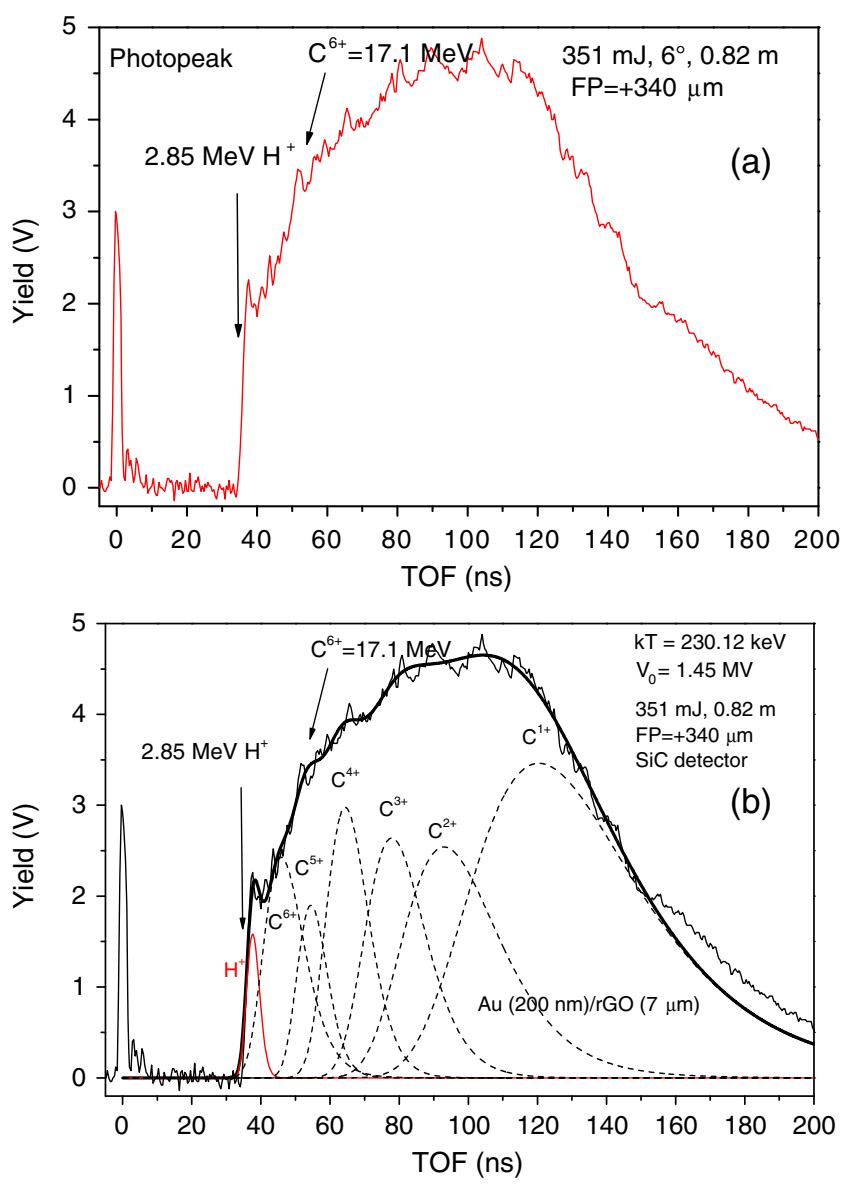

FIG. 3. SiC-TOF spectrum relative to the maximum ion acceleration (a) and peak deconvolution for protons and carbon ions using the CBS distribution function (b).

the detection of $4 \times 10^{-10} \mathrm{C}$. This charge is due to the collection of electrons generated in $\mathrm{SiC}$ by about $2 \mathrm{MeV}$ protons. Thus, by considering the $\mathrm{SiC}$ gap energy of $3.3 \mathrm{eV}$, the number of protons producing the TOF signal should be about $8.3 \times 10^{3}$ protons $/ 6 \mu$ str [10]. Now, due to the high directivity of the accelerated ions, assuming that the proton emission occurs on a solid angle of about $\pi / 2$ str, it means that the total proton emission should correspond to about $2.2 \times 10^{9}$ protons/pulse. With this assumption, the total energy transported by the protons is $0.7 \mathrm{~mJ}$ and the corresponding conversion efficiency of the laser in proton acceleration of $0.2 \%$.

Of course, the carbon emission is higher than this value and by the yield areas of the carbon ion signal, it is possible to evaluate as a first approximation that the total carbon emission should be of the order of 20 times higher than for protons, i.e., of about $4.4 \times 10^{10}$ carbon ions/pulse.

The reported TOF spectrum of Fig. 3 changes using a $\mathrm{SiC}$ detector covered by Mylar absorbers because ions, having a range lower than the Mylar thickness, are not detected. The spectra detected using the Mylar foil of 12 microns and 23 microns are reported in Fig. 4(a), 


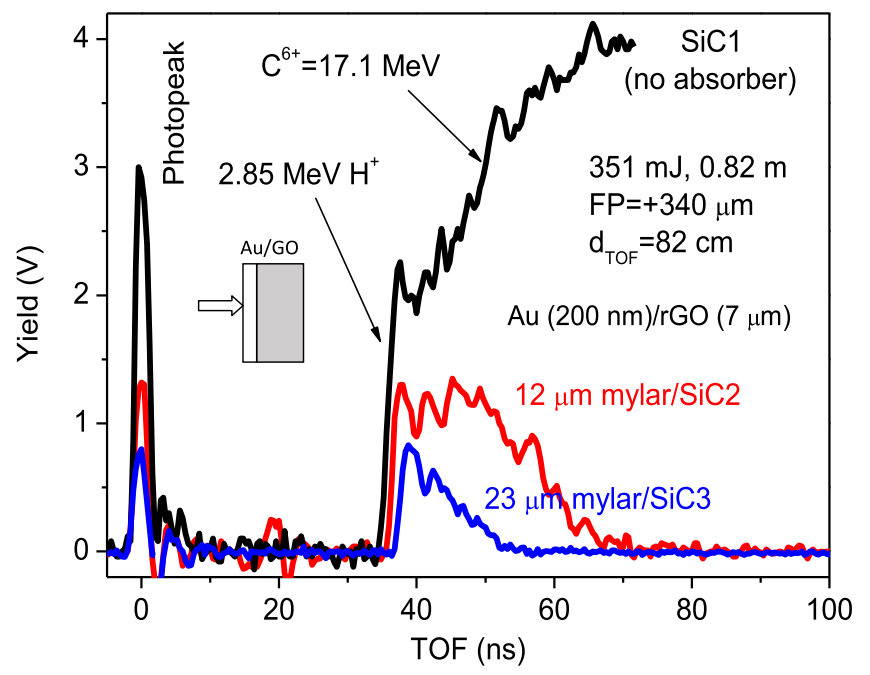

FIG. 4. Comparison of SiC-TOF spectra relative to the maximum ion acceleration with $\mathrm{SiC}$ without and with different thickness of Mylar absorber.

together with the spectrum relative to $\mathrm{SiC}$ without absorber for useful comparison. Spectra show that the proton peak is always present, in fact, the range of $2.85 \mathrm{MeV}$ protons in Mylar is 105 microns, higher than the thickness of both absorbers. The protons travel for the $0.82 \mathrm{~m}$ flight distance with the velocity corresponding to the $2.85 \mathrm{MeV}$ kinetic energy and are transmitted by the filter, thus they are not shifted in timescale. The $17.1 \mathrm{MeV}$ carbon ions have a range in Mylar of 19 microns, thus they are detected by the $\mathrm{SiC}$ using 12-microns thickness and not by the $\mathrm{SiC}$ using 23-microns absorber thickness, confirming the presence of $\mathrm{C}$ ions in the ion spectrum. The spectrum obtained using the 23 Mylar thickness as absorber indicates that the minimum proton energy is of about $1.4 \mathrm{MeV}$, an energy at which the range in Mylar is 33 microns. Thus, this analysis indicates that accelerated protons have a near monochromatic energy ranging between 2.85 and $1.4 \mathrm{MeV}$.

Figure 5 reports that, by irradiating the reverse foil, from the graphene oxide surface toward the gold film, and not from the Au surface, the proton acceleration decreases to $2.4 \mathrm{MeV}$ (a). By irradiating only the 7 microns $\mathrm{GO}$ without Au coverage the maximum proton energy decreases to $900 \mathrm{keV}$ (b). By irradiating only Au thin film (200 nm thickness) the maximum proton acceleration decreases to $700 \mathrm{keV}$ (c). Thus, the proton acceleration to $2.85 \mathrm{MeV}$ is the result of many advantages shown by the prepared thin foil. This target, in fact, increases the plasma density by the use of the Au surface thin film, uses a suitable thickness of the carbon foil with a very low density to enhance the transport of electrons in the rear side of the target, and uses high electrical and thermal conductivity of the foil. Moreover, an important factor of the high proton acceleration is due to the special conditions used for the focal position (FP) of the laser beam. The optimal conditions
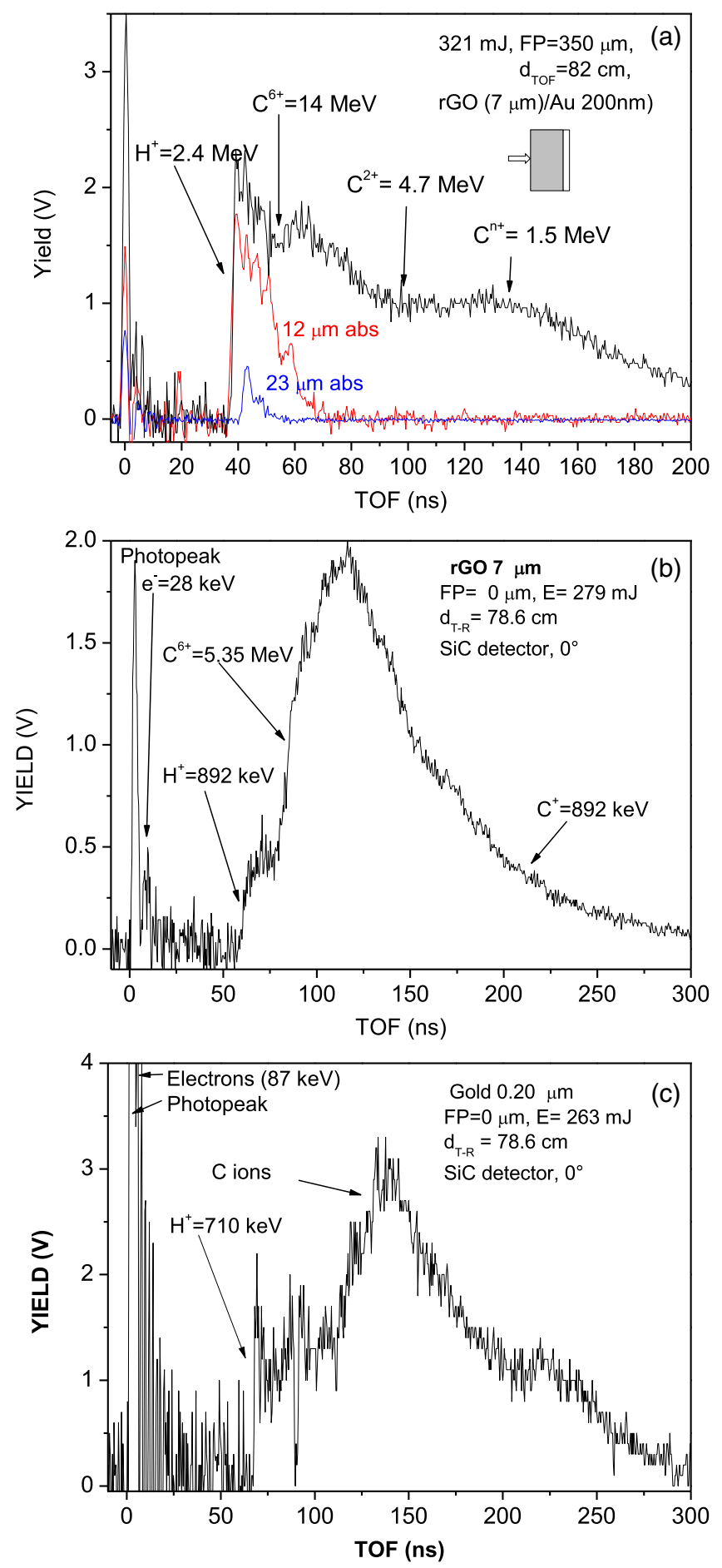

FIG. 5. SiC spectra obtained irradiating the sample from the GO side (a), irradiating the only GO foil without Au film (b) and irradiating the only $200 \mathrm{~nm}$ Au film without GO (c).

were found for $\mathrm{FP}=+340$ microns. By changing this FP the proton acceleration decreases. Measurements were performed in the range -400 microns (in front of the target surface), to 0 microns (at the Au target surface), and up to +400 microns (inside the target), confirming that the 

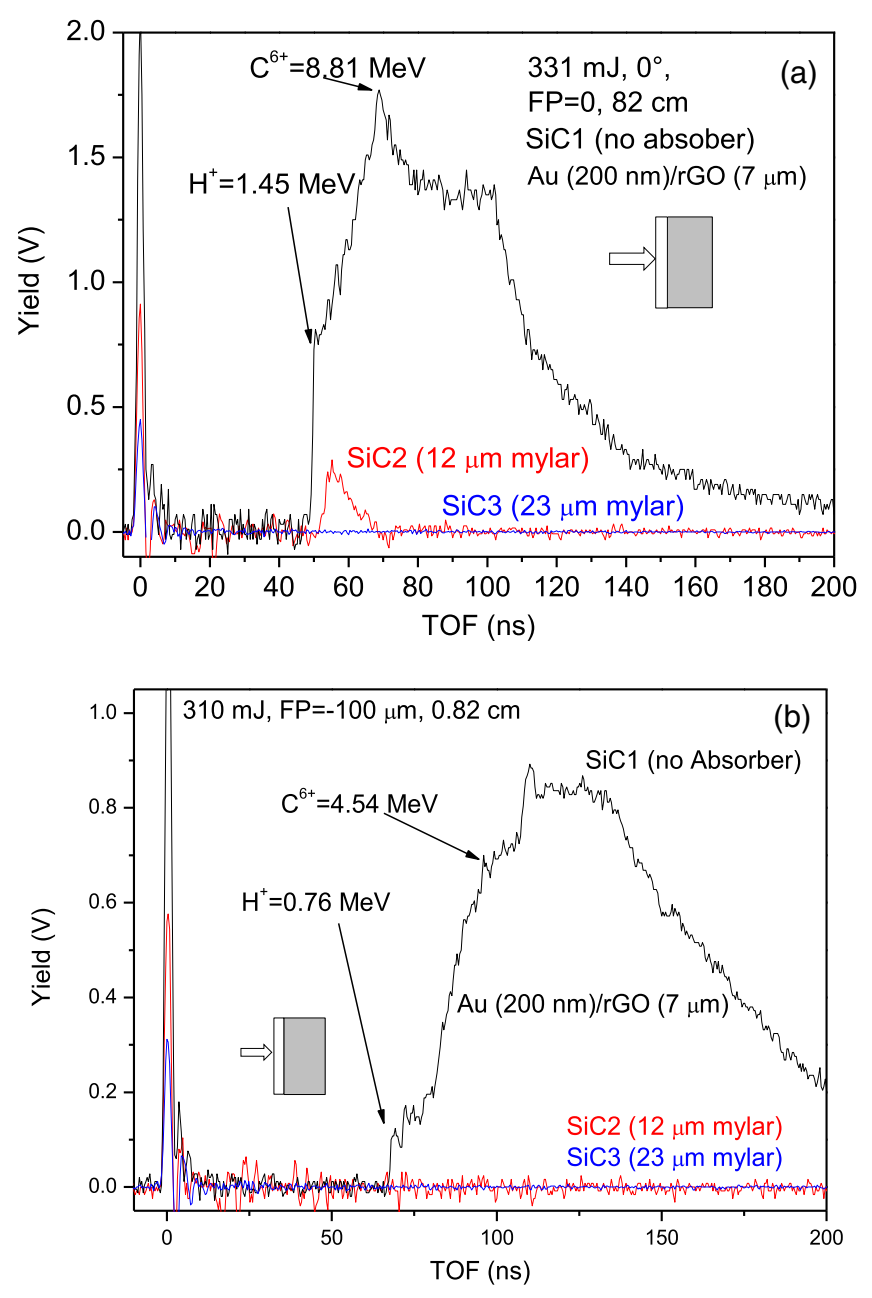

FIG. 6. SiC spectra obtained using a laser focal position of 0 microns (a) and of -100 microns (b).

maximum acceleration occurs near +340 microns. Figure 6 reports two $\mathrm{SiC}$ spectra of the $\mathrm{Au} / \mathrm{GO}$ foil obtained using an FP $=0 \mu \mathrm{m}$ (a) and FP $=-100 \mu \mathrm{m}$ (b) demonstrating that in these cases the maximum proton energy is of $1.4 \mathrm{MeV}$ and $760 \mathrm{keV}$, respectively. The total measurements performed by changing the focal position are reported in the plot of Fig. 7. The error on the proton energy measurement is of about $12 \%$, while the focal position error is of about $\pm 1 \mu \mathrm{m}$. It demonstrates that in general, the proton energy increases with the focal position inside the target. This effect increases the spot area and consequently, the number of electrons are accelerated forward by the incident laser pulse. Thus, electron density of the accelerating plasma increases and consequently the proton acceleration.

It is possible to note that two peaks occur in the proton energy plot. One less marked occurs at about $-25 \mu \mathrm{m}$, probably due to the self-focusing effect produced in front of the target [11], and another, more pronounced, occurs to $+340 \mu \mathrm{m}$ at which the electron density and plasma temperature have a maximum value, in agreement with similar

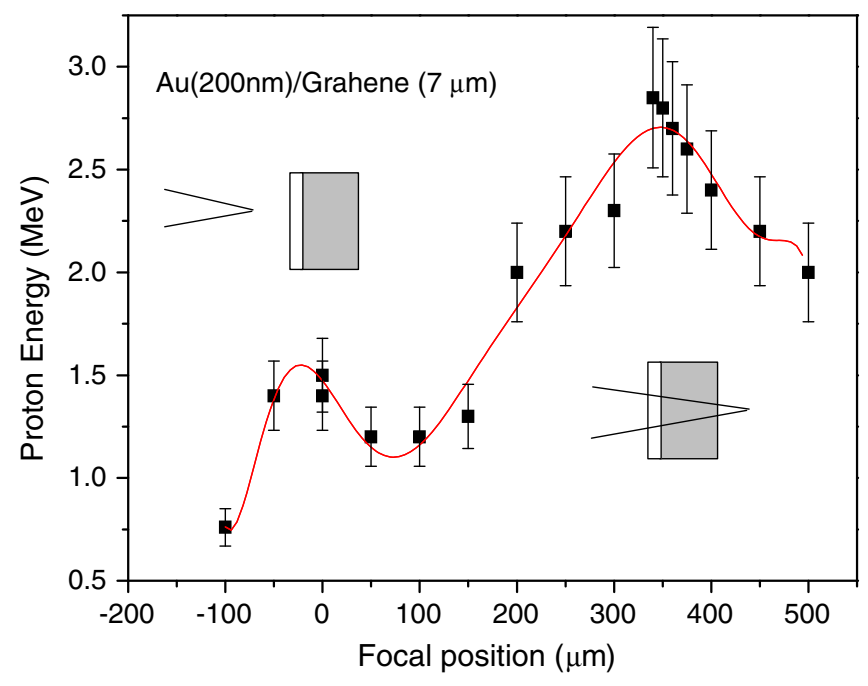

FIG. 7. Experimental maximum proton energy as a function of the laser focal position.

measurements present in literature by changing the focal position [12].

FP higher than the 340 microns decreases the ion acceleration effect because the laser intensity decreases for the high spot and the electron energy decreases, the electron transmitted to the rear side of the foil decreases, and consequently the acceleration as well.

\section{DISCUSSION AND CONCLUSIONS}

The relatively high proton energy obtained in this experiment is attributed to the special properties of GO foil, which has the advantages reported in Sec. II. The low GO density and low atomic number permit to transmit high relativistic electron density in the rear side of the foil enhancing the electric field responsible for the ion acceleration. In the case of used GO the graphene platelets [13] forming the GO foil, 7 microns in thickness, are randomly oriented, thus the real effect of electron channeling is minimum. In a next experiment, we want to improve the properties of GO using oriented platelets permitting higher electron channeling and low electron scattering so that we expect higher ion acceleration for these advanced targets to be demonstrated.

In any case, the use of these foils has permitted to generate high proton acceleration with significant proton yield and near-monochromatic energy, approximately within 2.85 and $1.4 \mathrm{MeV}$ and accelerating carbon ions with charge state from $1+$ up to $6+$ and kinetic energy of about $2.8 \mathrm{MeV} / \mathrm{z}$.

Measurements performed on pure GO, $7 \mu \mathrm{m}$ in thickness, or pure $\mathrm{Au}, 200 \mathrm{~nm}$ in thickness, have demonstrated that the proton acceleration is lower, thus the presence of both foils means that the target enhances the ion acceleration process, injecting electrons from the gold surface through the GO up to the rear side of the target. Similarly, 
the change of the laser focal position at +340 microns demonstrates the increment of the acceleration process, while other FP distances decrease it.

Although the obtained results are satisfactory, still much can be achieved both to increase the process of ion acceleration and that of the emitted ion stream, and of the energy conversion efficiency of the laser photons into the produced ion energy, which in these experiments generally is of the order of $10 \%$. Different improvements can be sought by using more suitable experimental conditions, such as to enhance the proton emission current enhancing a GO foil richer in absorbed hydrogen. The GO film thickness and the Au film thickness could be optimized to enhance the electron field acceleration, as reported in the literature. The structure of the GO could be used with higher crystallinity using oriented platelets to enhance the electron transmission of the foil. These are the directions of our investigations in the next experiments.

\section{ACKNOWLEDGMENTS}

This work was supported by the "Research and Mobility" project of Messina University No. 74893496, scientifically coordinated by Professor L. Torrisi. This work was also supported by the Grant Agency for the Czech Republic, project GACR “Center of Excellence”, N. P108/12/G108.

[1] M. Borghesi, Laser-driven ion acceleration: State of the art and emerging mechanisms, Nucl. Instrum. Methods Phys. Res., Sect. A 740, 6 (2014).

[2] M. Roth and M. Schollmeier, Ion acceleration-Target normal sheath acceleration, Proceedings CAS-CERN Accelerator School: Plasma Wake Acceleration, Geneva, Switzerland, 2014, edited by B. Holzer, CERN Report No. CERN-2016-001, Geneva, 2016, pp. 1-40.
[3] P. Mora, Plasma Expansion into a Vacuum, Phys. Rev. Lett. 90, 185002 (2003).

[4] L. Torrisi, Advanced polymer targets for TNSA regime producing $6 \mathrm{MeV}$ protons at $10^{16} \mathrm{~W} / \mathrm{cm}^{2}$ laser intensity, Phys. Plasmas 24, 023111 (2017).

[5] I. Pavlovsky and R. L. Fink, Graphene stripper foils, J. Vac. Sci. Technol. B 30, 03 D106 (2012).

[6] M. Aliofkhazraei, N. Ali, W. I. Milne, C. S. Ozkan, S. Mitura, and J. L. Gervasoni, Electrical and Optical Properties (CRC Press, Boca Raton, 2016).

[7] L. Torrisi, Large-scale studies of ion acceleration in lasergenerated plasma at intensities from $10^{10} \mathrm{~W} / \mathrm{cm}^{2}$ to $10^{19} \mathrm{~W} / \mathrm{cm}^{2}$, Opt. Laser Technol. 99, 7 (2018).

[8] J. Ziegler, SRIM-The Stopping and Range of Ions in Matter, http://www.srim.org/.

[9] L. Torrisi, Coulomb-Boltzmann-shifted distribution in laser-generated plasmas from $10^{10}$ up to $10^{19} \mathrm{~W} / \mathrm{cm}^{2}$ intensity, Radiat. Eff. Defects Solids 171, 34 (2016).

[10] M. Cutroneo, P. Musumeci, M. Zimbone, L. Torrisi, F. La Via, D. Margarone, A. Velyhan, J. Ullschmied, and L. Calcagno, High performance $\mathrm{SiC}$ detectors for $\mathrm{MeV}$ ion beams generated by intense pulsed laser plasmas, J. Mater. Res. 28, 87 (2013).

[11] L. Torrisi, D. Margarone, L. Laska, J. Krasa, A. Velyhan, M. Pfeifer, J. Ullschmied, and L. Ryc, Self-focusing effect in Au-target induced by high power pulsed laser at PALS, Laser Part. Beams 26, 379 (2008).

[12] L. Láska, J. Badziak, K. Jungwirth, M. Kálal, J. Krása, E. Krouský, P. Kubeš, D. Margarone, P. Parys, M. Pfeifer, K. Rohlena, M. Rosinski, L. Ryc, J. Skála, L. Torrisi, J. Ullschmied, A. Velyhan, and J. Wolowski, Analysis of processes participating during intense iodine-laser-beam interactions with laser-produced plasmas, Radiat. Eff. Defects Solids 165, 463 (2010).

[13] S. M. Shau, T. Y. Juang, H. S. Lin, C. L. Huang, C. F. Hsieh, J. Y. Wu, and R. J. Jeng, Individual graphene oxide platelets through direct molecular exfoliation with globular amphiphilic hyperbranched polymers, Polym. Chem. 3, 1249 (2012). 